\title{
Ambulanter Entzug mit Buprenorphin
}

\author{
Uwe Verthein \\ Michael Prinzleve \\ Peter Degkwitz \\ Georg Farnbacher \\ Michael Krausz
}

\section{Outpatient Detoxification Treatment with Buprenorphine}

\section{Zusammenfassung}

Studienziel: Im Rahmen einer bundesweit durchgeführten Anwendungsbeobachtung ${ }^{1}$ zur Behandlung Opiatabhängiger mit Buprenorphin wird untersucht, welche Patienten für eine ambulante Entzugsbehandlung erreicht werden und inwieweit sich Entzugssymptomatik und Konsumverhalten unter der Behandlung ändern. Design: Es handelt sich um eine prospektive naturalistische Studie über 12 Wochen. Erhebungen unter Ärzten und Patienten fanden zu Behandlungsbeginn, nach einer, 5, 9 und 12 Wochen statt. Setting: Die Studie wurde mit niedergelassenen Ärzten durchgeführt. Die Patienten wurden zwischen Februar 2000 und April 2001 rekrutiert. Untersuchungsgruppe: Einschlusskriterium waren ein Mindestalter von 18 Jahren und eine nachgewiesene Opiatabhängigkeit. Von 148 Patienten, die einen buprenorphingestützten Entzug durchführen wollten, liegen valide Daten zum Aufnahmezeitpunkt und Behandlungsverlauf vor. Variablen: Im Mittelpunkt der deskriptiven Analyse stehen Teilnahmedauer, Buprenorphindosis, Konsumverhalten und Entzugssymptomatik. Ergebnisse: $73 \%$ der Patienten schlossen die Studienbehandlung nach durchschnittlich 86 Tagen regulär ab. Bei 55\% lassen sich zum Ende der Entzugsbehandlung kein Methadon und keine weiteren Opiate mehr im Urin nachweisen. Es kommt zu einem signifikanten Rückgang der Entzugssymptomatik. Schlussfolgerungen: Der ambulante Entzug mit Buprenorphin durch niedergelassene Ärzte ist sicher durchführbar und kann für viele Patienten eine erfolgreiche Behandlungsoption darstellen.

\section{Schlüisselwörter}

Buprenorphin · Opiatabhängigkeit · ambulante Entzugsbehandlung $\cdot$ naturalistische Studie

\section{Abstract}

Study objective: In a nationwide postmarketing surveillance of opiate dependent patients treated with buprenorphine, the acceptance and efficacy regarding withdrawal symptoms and drug use of an outpatient drug detoxification treatment was examined. Design: The study was conceived as a prospective naturalistic study over 12 weeks, data were collected from doctors and patients at the onset of treatment and after one, 5, 9 and 12 weeks. Setting: The study was carried out with general practitioners. The patients were recruited between February 2000 and April 2001. Sample: Inclusion criteria were a minimum age of 18 years and a certified opiate dependency. Valid data from the time of inclusion and the course of treatment are available for 148 patients, who intended to undergo detoxification treatment based on buprenorphine. Variables: The descriptive analysis focuses on the length of participation, the buprenorphine dosage, consumption behaviour and withdrawal symptoms. Results: $73 \%$ of the patients regularly concluded the study treatment after an average of 86 days. In $55 \%$ of patients, no traces of methadone or other opiates can be detected in urine analyses at the conclusion of the detoxification treatment. Withdrawal symptoms significantly decrease. Conclusions: The outpatient detoxification treatment with buprenorphine by general practitioners can be a reliable, practicable and effective method for opiate dependent patients.

\section{Key words}

Buprenorphine - opiate dependence - outpatient detoxification · naturalistic study

\footnotetext{
${ }^{1}$ Die Studie wurde von ESSEX Pharma GmbH unterstützt.
} 
Vor dem Hintergrund eines mittlerweile relativ breiten Angebots von Substitutionsbehandlungen in Deutschland wurde im Februar 2000 mit subutex ${ }^{\circledR}$ (Buprenorphin) ein weiteres Medikament zur Substitutionstherapie Opiatabhängiger in Deutschland zugelassen. Mit insgesamt über 46000 Behandlungsplätzen gab es im Jahr 2000 für mindestens ein Drittel der Opiatabhängigen in Deutschland (ca. 120000 Personen) Angebote in der Substitutionsbehandlung [1]. Allerdings unterscheidet sich die Verbreitung der jeweils eingesetzten Substitutionsmedikamente erheblich. $69 \%$ aller Substituierten erhielten d,l-Methadon, dazu kommen $22 \%$, die mit Levomethadon behandelt wurden. $8 \%$ wurden mit Dihydrocodein substituiert und etwa 1000 Patienten erhielten Buprenorphin (1-2\%). Bezogen auf den Gesamtzeitraum des Jahres 2001 wurden ca. 7000 Patienten mit Buprenorphin behandelt.

Die Substitutionsbehandlung mit Methadonpräparaten gilt nach wie vor als Standardbehandlung für opiatabhängige Patienten. Allerdings zeigen Untersuchungen und Beobachtungen von Praktikern Einschränkungen bei der Anwendung von Methadon. Dabei wird vor allem auf das hohe Abhängigkeitspotenzial und die stärkere Entzugssymptomatik sowie auftretende Nebenwirkungen wie Müdigkeit, starkes Schwitzen und depressive Verstimmungen hingewiesen. Bezüglich dieser Begleiteffekte kann der Einsatz von Buprenorphin Vorteile bieten, da aufgrund seiner pharmakodynamischen Eigenschaften die von anderen Opioiden bekannten typischen (Neben-)Wirkungen teilweise ausbleiben.

Buprenorphin ist ein partieller Agonist am $\mu$-Opioid-Rezeptor und ein Antagonist am $\kappa$-Rezeptor und besitzt damit einen unter den Opioiden besonderen Wirkmechanismus. Über den $\mu$-Rezeptor werden Effekte wie Euphorie, Analgesie und Atemdepression vermittelt, wohingegen über den $\kappa$-Rezeptor Wirkungen wie Dysphorie und Sedierung erzeugt werden. Es gibt Hinweise darauf, dass aufgrund der antagonistischen Wirkung von Buprenorphin am $\kappa$-Rezeptor vor allem die unerwünschten psychischen Begleiteffekte in geringerem Maße auftreten.

Durch die sublinguale Applikationsform erfolgt initial eine schnelle Resorption. 60 bis 90 Minuten nach der Gabe werden maximale Plasmaspiegel erreicht. Aufgrund der langsamen Rückumverteilung aus dem Körpergewebe, die für konstante Plasmaspiegel sorgt, beträgt die Halbwertszeit etwa 20 Stunden und mehr. Bei nachträglicher Gabe von Heroin oder Methadon lässt sich Buprenorphin nur noch schwerlich aus der Rezeptorbindung verdrängen. Eine Wirkverstärkung im Sinne additiver Effekte ist damit unwahrscheinlich und die Gefahr einer Opioid-Überdosierung im Vergleich zu Methadon geringer einzuschätzen.

\section{Internationale Erfahrungen und Studien zum Einsatz von Buprenorphin}

Eine besonders breite Anwendung findet Buprenorphin in Frankreich. Laut Jahresbericht der EBDD (Europäische Beobachtungsstelle für Drogen und Drogensucht) gab es im Dezember 1999 bei geschätzten 160000 Opiatabhängigen 71260 Substituierte, von denen 62900 Buprenorphin und 8360 Methadon erhielten [2]. In Frankreich kann jeder niedergelassene Arzt Buprenorphin an Drogenabhängige verschreiben mit Take-home-Dosen von
$32 \mathrm{mg}$ für bis zu 28 Tage. Einerseits wird das starke Absinken der Anzahl drogenbedingter Todesfälle in den letzten Jahren in Frankreich mit der Einführung und Ausweitung der Buprenorphinbehandlung in Zusammenhang gebracht [3]. Andererseits wird, obwohl Buprenorphin auch dort als Sublingualtablette vorliegt, aufgrund der liberalen Vergabemodalitäten von häufigem Missbrauch gesprochen; zum einen aufgrund eines hohen Anteils von illegal verkauftem Buprenorphin, zum anderen durch intravenösen Konsum und Beikonsum von Benzodiazepinen [4].

In einer Studie von De Ducla et al. [5] zur Wirkung der Buprenorphinbehandlung mit 300 Patienten in 71 Zentren konnten positive Ergebnisse festgestellt werden. Die Behandlungsdauer lag zwischen 8 und 22 Monaten, die mittlere initiale Dosis betrug $6 \mathrm{mg}$ pro Tag, in der Stabilisierungsphase $8 \mathrm{mg}$. Die Einstellung des Heroinkonsums bei $70 \%$ der Patienten konnte allerdings nicht allein auf die Gabe von Buprenorphin zurückgeführt werden, vielmehr wurden als Bedingungen zur Verbesserung der Behandlung Schulungsmaßnahmen für niedergelassene Ärzte und Apotheker für erforderlich gehalten sowie eine bessere Koordination mit anderen Bereichen des Suchthilfesystems.

Wenngleich Buprenorphin bereits seit knapp 40 Jahren Gegenstand der Forschung ist [6], liegen umfangreiche Vergleichsstudien zwischen Buprenorphin und anderen Substitutionsmitteln erst seit Anfang der 90er-Jahre vor [7-13]. Die erzielten Ergebnisse lassen allerdings keine eindeutigen Schlüsse hinsichtlich der Überlegenheit der einen oder anderen Substanz zu. So sind sowohl die in den Studien zu vergleichenden Dosierungen als auch die Präparate selbst sehr unterschiedlich. Auch die Aufdosierungen von Buprenorphin bei der Einstellung werden verschieden gehandhabt. Darüber hinaus sind die Untersuchungen in unterschiedlichen Behandlungssettings - ambulant, stationär, in speziellen Ambulanzen, Entgiftungsstationen, bei niedergelassenen Ärzten - durchgeführt worden.

Die Metaanalysen von Barnett et al. [14] und West et al. [15] kommen zu dem Ergebnis, dass Buprenorphin als effektive Alternative zur traditionellen Methadonerhaltungstherapie angesehen werden kann. Aufgrund der Unterschiede in den Studiendesigns sowie der unterschiedlichen Dosierungen (vor allem von Methadon) bleiben jedoch viele Fragen offen. Dies betrifft v. a. die Dosisabhängigkeit der Effekte, die Vorerfahrungen der Patienten mit Substitutionsbehandlungen sowie die Rolle der psychosozialen Begleitung, die in den meisten Studien nur unzureichend untersucht wurde.

Petitjean et al. [16] untersuchten Sicherheit, Effektivität und Praktikabilität von Buprenorphin im Vergleich zu Methadon in einer randomisierten Doppelblindstudie. Diese Untersuchung war die erste klinische Studie, die Buprenorphin in seiner sublingualen Form mit oralem Methadon verglich. Im Ergebnis schlossen $56 \%$ der Buprenorphingruppe die Behandlung regulär ab, bei der Methadongruppe waren es $90 \%$. Nahezu alle Abbrüche in der Buprenorphingruppe fanden in den ersten Tagen statt. Von den Abbrechern klagten $67 \%$ über Entzugserscheinungen, was möglicherweise auf die mit $4 \mathrm{mg}$ Buprenorphin sehr geringe Einstiegsdosis zurückzuführen sein könnte. In den über die Dauer von sechs Wochen durchgeführten Urinkontrollen unterschieden sich die beiden Gruppen kaum: Die Anteile opiatpositiver Proben 
waren vergleichbar (Buprenorphin: 62\%, Methadon: 60\%). Ebenfalls keine signifikanten Unterschiede gab es beim Kokain. Auch im Verlangen (Craving) nach Heroin unterschieden sich die beiden Gruppen nicht. Eine Gleichwertigkeit von Buprenorphin und Methadon hinsichtlich der Haltequote und der Reduzierung des Opiatkonsums stellten auch Pani et al. [17] in ihrer Wirksamkeitsstudie mit Sublingualtabletten fest. Es kam zwar zu einer tendenziell höheren Abbrecherquote in der Buprenorphingruppe, dies wird aber wiederum mit einer möglicherweise zu geringen Einstellungsdosis sowie mit der geringeren Bioverfügbarkeit der Tablette gegenüber der flüssigen Form begründet.

Der ambulante Entzug mit Buprenorphin stand im Mittelpunkt der Studie von Diamant et al. [18]. Im Rahmen eines 10-tägigen Abdosierungsprozesses mit täglicher Dosisreduktion konnte bei den 50 einbezogenen Patienten eine Compliance von $70 \%$ erreicht werden. Die Entzugssymptomatik, gemessen mit der Wang-Skala [19], verringerte sich deutlich von durchschnittlich 13,8 am ersten Tag auf 5,4 Punkte am 10. Tag. Unter den Patienten, die die Entzugsbehandlung bis zum 10. Tag durchhielten, hatten am Ende 69\% opiatnegative Urinproben. Resnick et al. [20] untersuchten in einem dreijährigen Projekt mit niedergelassenen Ärzten den Einsatz von Buprenorphin bei Abhängigen, die einer Methadonbehandlung nicht zugänglich waren. Auch bei diesen Patienten war eine ambulante Entzugsbehandlung mit Buprenorphin möglich. Es kam zu einem deutlichen Rückgang des Heroin-Craving und die Patienten zeigten beim Absetzen des Buprenorphins nur noch geringe Entzugssymptome.

Die Verwendung von Buprenorphin im Rahmen eines stationären Entzugs über 10 Tage wurde von Vignau [21] berichtet. Ein Viertel der Patienten wechselte während der Entzugsbehandlung in ein methadongestütztes Setting, $58 \%$ beendeten den Entzug planmäßig. Bereits am 4. Tag hatten alle Patienten keine opiatpositiven Urinproben mehr. Auch in einer bundesdeutschen Kontrollgruppenstudie wurde der Einsatz von Buprenorphin (in Kombination mit Carbamazepin) in der stationären Entzugsbehandlung im Vergleich zu einer Oxazepam/Carbamazepin-Behandlung untersucht [22]. Unter den Buprenorphinpatienten brachen $27 \%$ die Behandlung vor dem 21. Tag ab, die Abbruchquote bei den Kontrollpatienten lag bei $42 \%$. Wenngleich die Studienergebnisse nur auf einer Stichprobe von $n=27$ Patienten beruhen (Experimentalgruppe: $n=15$, Kontrollgruppe: $\mathrm{n}=12$ ), erwies sich die kombinierte Buprenorphin/Carbamazepin-Behandlung hinsichtlich der Reduktion von Entzugssymptomen als signifikant wirkungsvoller.

Insgesamt deuten die Ergebnisse zum Einsatz von sublingualem Buprenorphin sowohl in der Substitutionsbehandlung als auch in der Entgiftung auf positive Wirkungen hin. Kontrollierte Studien zur Vergleichbarkeit mit anderen Substitutionsmitteln lassen jedoch noch keine eindeutigen Schlüsse hinsichtlich der Überlegenheit von Buprenorphin zu. In Verbindung mit der Einführung von Buprenorphin zur Behandlung Opiatabhängiger in Deutschland dürften somit zunächst Fragen nach Erreichbarkeit, Compliance und Möglichkeiten des Einsatzes von Buprenorphin im Rahmen unterschiedlicher Settings (niedergelassene Ärzte, Ambulanzen) und Behandlungsziele (Erhaltungstherapie, Entzugsbehandlung) stehen.

\section{Fragestellung}

Die Verwendung von Buprenorphin ${ }^{2}$ zur ambulanten Entzugsbehandlung Opiatabhängiger durch niedergelassene Ärzte soll geprüft werden. Folgende Fragestellungen stehen im Zentrum der Untersuchung:

- Welche Opiatabhängigen werden von der Entzugsbehandlung mit Buprenorphin erreicht bzw. vornehmlich angesprochen?

- Wie hoch ist die Haltequote? Wie gestaltet sich der Behandlungsverlauf?

- In welchem Umfang ändern sich Entzugssymptomatik und Konsumverhalten?

\section{Methode}

Der Einsatz von Buprenorphin in der ambulanten Entzugsbehandlung wurde im Rahmen einer Anwendungsbeobachtung [23], die als prospektive Verlaufsstudie über zwölf Wochen durchgeführt wurde, untersucht. In acht über die Bundesrepublik Deutschland verteilten Regionen nahmen 215 Ärzte an der Studie teil. Insgesamt 427 Patienten wurden im Zeitraum von Februar 2000 bis April 2001 rekrutiert. Die Einschlusskriterien orientierten sich an der zusammenfassenden Fachinformation der Merkmale des Arzneimittels (SPC) und bestanden in einem Mindestalter von 18 Jahren und einer nachgewiesenen Opiatabhängigkeit. Ausschlusskriterien waren die Nichtverträglichkeit von Buprenorphin, schwere respiratorische Insuffizienz, schwere Leberinsuffizienz, Alkoholabhängigkeit, eine Behandlung mit Monoaminooxidasehemmern sowie das Stillen von Säuglingen. Ein- und Ausschlusskriterien wurden vom behandelnden Arzt beurteilt und überprüft. Zur Zielgruppe zählten Patienten mit mittlerem Abhängigkeitsniveau, bisher nicht erfolgreicher Substitutionsbehandlung, Entzugsvorsatz, psychischer Vulnerabilität und/oder Bedarf an einer Übergangsbehandlung bis zur Aufnahme einer anderen Suchttherapie.

\section{Studiendesign}

Die Veränderungen im Verlauf der Entzugsbehandlung wurden parallel aus der Perspektive der behandelnden Ärzte und der Patienten anhand standardisierter Fragebogen untersucht. Zentrale Verlaufskriterien waren die Entzugserscheinungen und das Konsumverhalten. Zu Beginn der Studie wurden der Behandlungsanlass, die Rahmenbedingungen, Angaben zum Gesundheitszustand, das Ergebnis des letzten Urinscreenings sowie, bei bereits substituierten Patienten, Daten zur bisherigen Substitutionsbehandlung in einem Aufnahmebogen dokumentiert. Zudem wurde den Patienten bei Behandlungsbeginn ein Fragebogen vorgelegt, in dem Daten zur sozialen Situation, zum Drogenkonsum, zur Entzugssymptomatik, zum psychischen Befinden (Depressivität anhand des BDI [24], Ängstlichkeit mit dem STAI [25]) sowie zu Nebenwirkungen des bisherigen Substitutionsmittels erhoben wurden. Hinsichtlich des Drogenkonsums wurden die aktuelle Konsumintensität, d.h. die Anzahl an Konsumtagen innerhalb der letzten 30 Tage, die Hauptproblemdroge sowie das Einstiegsalter beim Heroin- und Kokainkonsum

\footnotetext{
${ }^{2}$ In der vorliegenden Studie wurde Buprenorphin ausschließlich in der sublingualen Form als subutex ${ }^{\circledR}$ verwendet.
} 
und die Behandlungsvorerfahrungen erfragt. Die Ausprägung der Entzugssymptomatik wurde anhand der Short Opiate Withdrawal Scale SOWS [26] erhoben.

Die individuellen Verläufe wurden nach der Ausgangserhebung $\left(\mathrm{T}_{0}\right)$ zu Behandlungsbeginn an vier weiteren Erhebungszeitpunkten dokumentiert: in der ersten $\left(T_{1}\right)$, fünften $\left(T_{2}\right)$, neunten $\left(T_{3}\right)$ und zwölften $\left(\mathrm{T}_{4}\right)$ Woche der Studie. Die Erhebung des Konsumverhaltens und des psychischen Befindens bei den Patienten fand nur in der zwölften Woche $\left(\mathrm{T}_{4}\right)$, d.h. in der Abschlusserhebung, statt. In den Fällen, in denen die Studie vorzeitig beendet oder abgebrochen wurde, wurde von den behandelnden Ärzten der bei regulärem Ende $\left(\mathrm{zu} \mathrm{T}_{4}\right)$ vorgesehene Abschlussbogen ausgefüllt. Bei $\mathrm{T}_{4}$ handelt es sich daher um den jeweils letzten $\mathrm{Er}$ hebungszeitpunkt $\left(\mathrm{T}_{4 / \mathrm{lzt}}\right)$, der nur bei vollständigem Durchlaufen der Studie in der zwölften Woche lag. Die Behandlungsdauer variiert somit für alle Patienten, von denen ein Abschlussbogen vorliegt.

\section{Untersuchungsgruppe}

Die Daten der Fragebogen wurden einer Analyse der missing data und Plausibilitätsprüfungen unterzogen, die Patienten-IDs wurden auf Unstimmigkeiten und Doppelungen überprüft. Als Ergebnis dieser Datenbereinigung mussten die Datensätze von 37 der 427 Teilnehmer von der weiteren Auswertung ausgeschlossen werden. Für die Auswertung standen somit die Datensätze von 390 Teilnehmern zur Verfügung, von denen die ärztlichen Aufnahmebogen vorliegen. Die ebenfalls bei Behandlungsbeginn vorgelegten Patientenfragebogen füllten jedoch nur 314 Teilnehmer (81\% von 390$)$ aus [23].

Bei $47 \%$ dieser Patientengruppe $(n=148)$ wurde ein ambulanter Entzug durchgeführt. Von nur 92 Patienten, entsprechend $62 \%$, liegen Informationen zu den wichtigsten Verlaufsvariablen zum letzten Erhebungszeitpunkt ( $\left.\mathrm{T}_{4 / \mathrm{zz}}\right)$ vor (Tab.1).

Tab. 1 Stichprobengrößen zu Behandlungsbeginn $\left(T_{0}\right)$ und Studienende $\left(T_{4 / \mid z t}\right)$, durchschnittlicher Zeitraum zwischen den Erhebungszeitpunkten

\begin{tabular}{lccc}
\hline & $\boldsymbol{T}_{\mathbf{0}}$ & $\boldsymbol{T}_{\mathbf{4 / z t}}$ & Zeitspanne \\
\hline ärztliche Untersuchung & 148 & 141 & 81,7 Tage \\
\hline Urinkontrollen & 136 & 114 & 84,7 Tage \\
\hline Patientenfragebogen & 147 & 92 & 89,4 Tage \\
\hline
\end{tabular}

Werden die Entzugspatienten ( $\mathrm{n}=148$ ) mit den übrigen Patienten, die eine Buprenorphinerhaltungs- oder Überbrückungstherapie erhielten $(n=166)$, bezüglich allgemeiner Merkmale und Behandlungsdaten zu Therapiebeginn verglichen, zeigen sich keine signifikanten Unterschiede. Erwähnenswert ist lediglich, dass der Anteil an Patienten, die direkt aus einer Substitutionsbehandlung kommen, sowie der Anteil an Selbstzahlern unter den Entzugspatienten etwas höher ist (Tab. 2).

Für knapp ein Drittel der Entzugspatienten war der Beginn der Buprenorphinbehandlung gleichbedeutend mit einem Einstieg (oder Wiedereinstieg) in die ambulante Substitutionsbehand-
Tab. 2 Vergleich zwischen Entzugspatienten und Patienten mit Erhaltungstherapie („Restgruppe“) hinsichtlich Geschlecht, Alter und Behandlungsdaten

\begin{tabular}{|c|c|c|}
\hline & Entzugspatienten & Restgruppe \\
\hline \multicolumn{3}{|l|}{ Geschlecht } \\
\hline männlich & $66 \%$ & $63 \%$ \\
\hline weiblich & $34 \%$ & $37 \%$ \\
\hline Durchschnittsalter, Jahre & $32,0( \pm 7,5)$ & $31,7( \pm 7,4)$ \\
\hline bisherige Substitution & $69 \%$ & $59 \%$ \\
\hline Substitutionsdauer, Monate & $28,3( \pm 26,0)$ & $25,7( \pm 26,2)$ \\
\hline \multicolumn{3}{|l|}{ Substitutionsmittel } \\
\hline d,I-Methadon & $47 \%$ & $37 \%$ \\
\hline Levomethadon & $18 \%$ & $13 \%$ \\
\hline Dihydrocodein & $2 \%$ & $5 \%$ \\
\hline keine Angaben & $2 \%$ & $3 \%$ \\
\hline \multicolumn{3}{|l|}{ Hauptgrund für Behandlung } \\
\hline mittleres/niedriges Abhängigkeitsniveau & - & $33 \%$ \\
\hline bisherige Substitution nicht erfolgreich & - & $22 \%$ \\
\hline geplanter Entzug mit Eigenmotivation & $100 \%$ & - \\
\hline psychische Auffälligkeit & - & $21 \%$ \\
\hline Überbrückungssubstitution & - & $8 \%$ \\
\hline Sonstiges & - & $15 \%$ \\
\hline \multicolumn{3}{|l|}{ Finanzierung } \\
\hline AUB/BUB (KK) & $58 \%$ & $68 \%$ \\
\hline Selbstzahler & $31 \%$ & $23 \%$ \\
\hline Sozialamt & $10 \%$ & $8 \%$ \\
\hline $\mathrm{n}$ & 148 & 166 \\
\hline
\end{tabular}

lung. $69 \%$ wurden bereits substituiert, wobei die Dauer der bisherigen Substitutionsbehandlung zwischen 2 Wochen und 10 Jahren variierte. Dabei wurde die Mehrheit der auf Buprenorphin umgestiegenen Patienten zuvor mit Methadon substituiert, 18\% mit Levomethadon.

Im Alter von 20,8 Jahren begannen die Patienten, regelmäßig Heroin zu konsumieren. Etwa zur gleichen Zeit (mit durchschnittlich 21,1 Jahren) erfolgte der Einstieg in den regelmäßigen Kokainkonsum. Die soziale Lebenssituation der Entzugspatienten zu Behandlungsbeginn stellte sich insgesamt - im Vergleich zur Situation der Opiatabhängigen in Deutschland [27] - sehr stabil dar (Tab. 3). Die Mehrheit lebte (mit Partner, Kindern oder allein) in einer eigenen Wohnung. Lediglich 2 Personen lebten zu Studienbeginn in Gelegenheitsunterkünften oder waren obdachlos (1\%). Insbesondere die Erwerbssituation war in der Untersuchungsgruppe vergleichsweise gut. Mit insgesamt $62 \%$ waren fast zwei Drittel der Patienten in einer regelmäßigen (Teilzeit-)Beschäftigung oder in einer Ausbildung.

\section{Ergebnisse}

Aus den Angaben der Ärzte ergibt sich, dass drei Viertel der Patienten die Entzugsbehandlung regulär abschlossen (73\%). Zu einem vorzeitigen Abbruch kam es bei $22 \%$, bei weiteren $5 \%$, bei denen die diesbezüglichen Angaben fehlen, ist von einem Behandlungsabbruch auszugehen. Insgesamt nahmen die Teilnehmer im Durchschnitt 75,8 $( \pm 32,3)$ Tage, also fast elf Wochen an 
Tab. 3 Die soziale Situation der Entzugspatienten bei Studienbeginn $(n=148)$

\section{Anteil in \%}

\begin{tabular}{|c|c|}
\hline \multicolumn{2}{|l|}{ Partnerschaft } \\
\hline allein stehend & 30 \\
\hline zeitweilige Beziehungen & 9 \\
\hline feste Beziehung, getrennt lebend & 16 \\
\hline feste Beziehung, zusammen lebend & 43 \\
\hline keine Angaben & 3 \\
\hline \multicolumn{2}{|l|}{ Kinder } \\
\hline ja & 40 \\
\hline nein & 56 \\
\hline keine Angaben & 4 \\
\hline \multicolumn{2}{|l|}{ Wohnsituation } \\
\hline mit Partner und/oder Kindern & 45 \\
\hline bei Eltern/Familienangehörigen & 16 \\
\hline mit Freunden/in WG & 5 \\
\hline allein, in eigener Wohnung & 32 \\
\hline keine feste Wohnsituation/obdachlos & 1 \\
\hline keine Angaben & 1 \\
\hline \multicolumn{2}{|l|}{ Erwerbssituation } \\
\hline ganztags tätig & 39 \\
\hline regelmäßige Teilzeitarbeit & 9 \\
\hline unregelmäßige Teilzeitarbeit & 5 \\
\hline Schüler, Student, Azubi & 9 \\
\hline (Früh-)Rentner & 2 \\
\hline arbeitslos & 26 \\
\hline in Institution & - \\
\hline Hausfrau, Hausmann & 6 \\
\hline keine Angaben & 3 \\
\hline
\end{tabular}

der Studie teil $(\mathrm{n}=104)$. Die regulären Beender blieben durchschnittlich 86,2 $( \pm 26,7)$ Tage in der Studie, die Abbrecher nur $46,1( \pm 28,5)$ Tage. Der Großteil der abbrechenden Patienten schied bereits in der ersten Woche aus der Studie aus, bzw. es lagen keine Informationen über den Status oder die Behandlungsaufnahme vor (Abb.1).

Von 24 Teilnehmern, die die Entzugsbehandlung abbrachen (entsprechend $60 \%$ aller Abbrecher), liegen Angaben zu den Gründen vor. Am häufigsten wurde ein Abbruch auf Wunsch

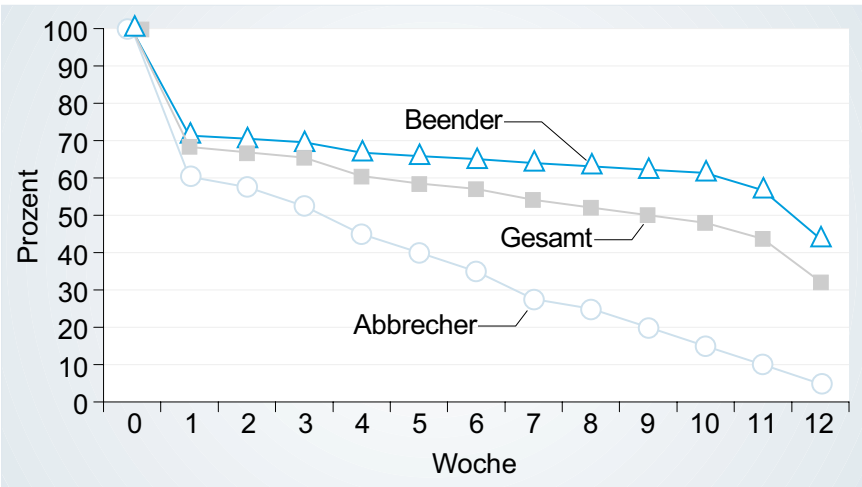

Abb. 1 Teilnahmedauer an der Entzugsbehandlung in Wochen - Haltequote $(n=148)$. des Teilnehmers (42\%) bzw. aufgrund des Nichterscheinens (33\%) angegeben. Eine unerwünschte Wirkung des Buprenorphins lag bei keinem Patienten als Abbruchgrund vor. Bei 38\% der Teilnehmer spielte unerlaubter Beigebrauch eine Rolle, die Behandlung abzubrechen.

\section{Dosierungsverlauf}

Bei der Beschreibung des Dosierungsverhaltens muss zwischen Patienten, die die Entzugsbehandlung regulär beendeten bzw. deren Behandlung einen regulären Verlauf nahm, und den Abbrechern unterschieden werden. Diese unterschiedlichen Behandlungsverläufe spiegeln sich im Dosierungsprozess wider (Abb. 2). Es fällt auf, dass bereits die Eingangsdosis der regulären Beender mit durchschnittlich 6,6 mg deutlich höher ist als die der (späteren) Abbrecher $(4,8 \mathrm{mg}$, t-Test: $\mathrm{t}=2,7, \mathrm{p}<0,01)$. Im Verlauf der ersten Woche $\left(T_{1}\right)$ stieg die Buprenorphindosis in beiden Gruppen leicht an, um im weiteren Behandlungsverlauf bis Woche $12\left(\mathrm{~T}_{4 / \mathrm{lzt}}\right)$ auf etwa $60 \%$ des Ausgangswerts abzusinken. Auch hier liegt die Durchschnittsdosis der Beender $(4,1 \mathrm{mg})$ wiederum signifikant über der der Abbrecher (2,8 mg, t-Test: $\mathrm{t}=2,1, \mathrm{p}<0,05)$.

\section{Behandlungseffekte}

Für die Auswertung des Drogenkonsums standen zum einen die Urinkontrollen (UK) und zum anderen die Selbstauskünfte der Patienten zur Verfügung. Wie aus Tab. 1 ersichtlich ist, liegen allerdings nicht von allen Patienten Informationen zum Zeitpunkt des Behandlungsendes $\left(\mathrm{T}_{4}\right)$ vor: 92 Patienten haben den Erhebungsbogen ausgefüllt, von 114 Personen liegen die Ergebnisse der Urinproben vor.

Im Mittelpunkt der Buprenorphinbehandlung steht der Entzug von Opioiden. Anhand der Urinkontrollen zu $\mathrm{T}_{4}$ nahmen $72 \%$ der Patienten zum Ende der Entzugsbehandlung (innerhalb des 12-wöchigen Untersuchungszeitraums) kein Heroin, Methadon oder andere Opiate mehr. Bezogen auf alle 148 Patienten sind dies immer noch $55 \%$. Bei den Opiaten zeigt sich ein Verlauf, der zum einen das Ergebnis der Selbstauskünfte hinsichtlich des deutlichen Rückgangs der Konsumprävalenz bestätigt (siehe unten). Zum anderen ergibt sich, dass dieser Rückgang sehr schnell einsetzte, bereits zu $\mathrm{T}_{1}$ sank der Anteil opiatpositiver UK-Ergebnisse von 43 auf $20 \%$. Dieser Anteil verringerte sich weiter auf $14 \%$ und stabilisierte sich auf diesem Niveau (Abb. 3). Ferner ist eine leichte Abnahme beim Nachweis von Benzodiazepinen und

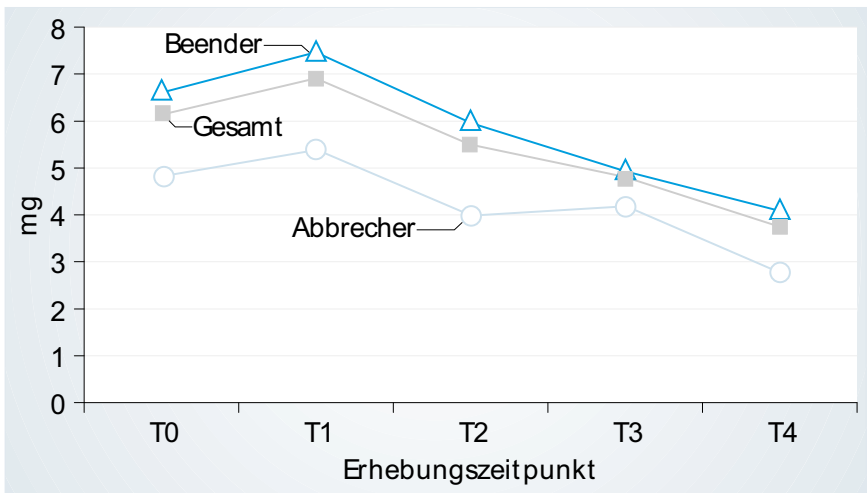

Abb. 2 Dosierung von Buprenorphin in Abhängigkeit vom Behandlungsstatus. 


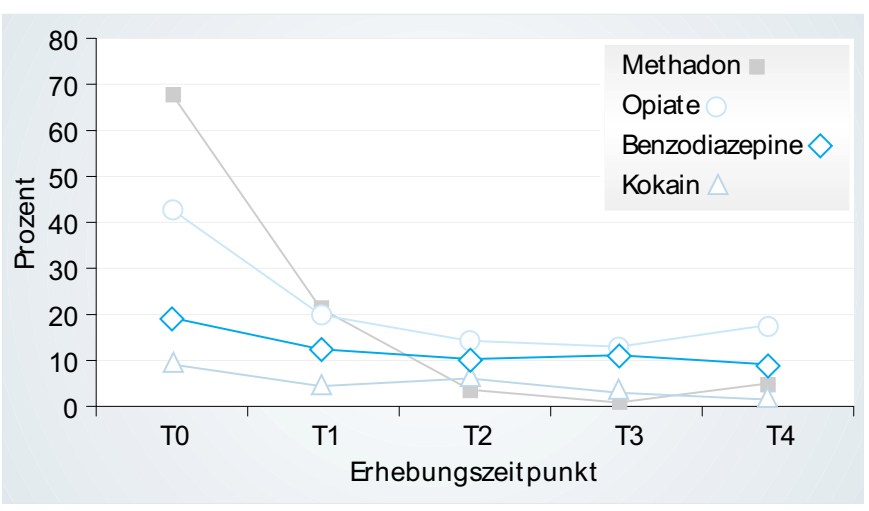

Abb. 3 Anteil positiver UK-Ergebnisse von Methadon, Opiaten, Benzodiazepinen und Kokain zu allen Erhebungszeitpunkten. McNemarTest zwischen $T_{0}$ und $T_{4 / 1 z t}$ : Methadon: $\chi^{2}=50,4, p<0.001, n=90$; Opiate: $\chi^{2}=17,4, p<0.001, n=104$; Benzodiazepine: Binomial, n.s., $\mathrm{n}=94$; Kokain: Binomial, n. s., $\mathrm{n}=96$.

Kokain zu beobachten, die allerdings keine statistische Signifikanz aufweist. Deutlich erkennbar ist der Rückgang an Methadon-positiven Urinproben, der den Umstieg auf Buprenorphin im Rahmen der Entzugsbehandlung aufzeigt.

Werden die Selbstauskünfte der Patienten zum Drogen- und Alkoholkonsum dargestellt, d.h. die Anzahl an Tagen mit Konsum in den letzten 30 Tagen, die bei Beginn und am Ende der Studie erhoben wurden, ergibt sich folgendes Bild: Es zeigt sich ein deutlicher Rückgang der Prävalenzrate für Heroin (Tab. 4, linke Hälfte). Während bei Behandlungsbeginn mehr als die Hälfte der Patienten Heroin konsumierte, waren es am Ende der Untersuchung noch $16 \%$. Dementsprechend gab es einen signifikanten Rückgang des intravenösen Drogengebrauchs von 31 auf $9 \%$. Auch der Konsum von nicht verschriebenem Methadon ging deutlich zurück (von 13 auf $1 \%$ ).
Bei der Betrachtung der Konsumintensität bzw. -frequenz, also der Anzahl an Tagen mit Konsum, ergibt sich ein ähnliches Bild (Tab. 4, rechte Hälfte). Auch hier sind es v. a. die Intensitäten von Heroin, nicht verschriebenem Methadon und intravenösem Konsum, die sich im Durchschnitt signifikant verringerten. Die ohnehin sehr geringen Konsumfrequenzen von Benzodiazepinen und Barbituraten, Amphetaminen und Halluzinogenen blieben im Wesentlichen unverändert.

Entscheidend für den Verlauf der Entzugsbehandlung ist die Bekämpfung von Entzugssymptomen. Abb. 4 zeigt den Verlauf der Entzugssymptomatik anhand der SOWS sowie aus ärztlicher Sicht. Dabei ist zu beachten, dass statt des SOWS-Gesamtscores der Mittelwert für die Darstellung berücksichtigt wird. Dieser kann Werte zwischen 0 und 3 annehmen und lässt sich daher gemeinsam auf einer Skala mit den ärztlichen Einschätzungen abbilden. Die Entzugssymptomatik war in der Untersuchungsgruppe bereits bei Behandlungsbeginn relativ gering ausgeprägt. Dennoch ergab sich bei der Betrachtung der SOWS-Mittelwerte

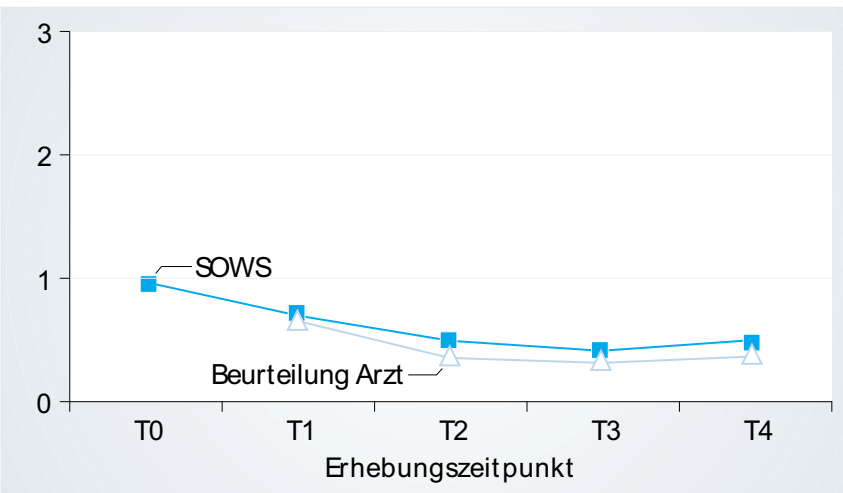

Abb. 4 Verlauf der Entzugssymptomatik aus Patientensicht (SOWS) und aus ärztlicher Sicht. Ratingskala Entzugssymptomatik: 0 = „gar keine“, 1 = „etwas“, 2 = „mäßig“, 3 = „stark“.

Tab. 4 Konsumverhalten bei Behandlungsbeginn und zum Zeitpunkt der Abschlussuntersuchung (Konsum innerhalb der letzten 30 Tage, Patientenangaben, $\left.T_{0}: n=147, T_{4 / 1 z t}: n=92\right)$

\begin{tabular}{|c|c|c|c|c|c|c|}
\hline & \multicolumn{2}{|c|}{ Prävalenz } & \multirow[b]{2}{*}{ Sign. ${ }^{1)}$} & \multicolumn{2}{|c|}{ Intensität (Konsumtage) } & \multirow[b]{2}{*}{ Sign. ${ }^{2)}$} \\
\hline & $T_{0}$ & $T_{4 / 1 / z t}$ & & $T_{0}$ & $T_{4 / / z t}$ & \\
\hline Alkohol jegl. Konsum & $55 \%$ & $36 \%$ & n. s. & $5,1( \pm 8,0)$ & $5,2( \pm 7,9)$ & n. s. \\
\hline Heroin & $51 \%$ & $16 \%$ & $\chi^{2}=13,8 * *$ & $9,0( \pm 12,0)$ & $1,7( \pm 4,4)$ & $t=5,2 * *$ \\
\hline Schwarzmarkt-Methadon & $13 \%$ & $1 \%$ & 3) * & $0,7( \pm 3,0)$ & $0,1( \pm 0,4)$ & $t=1,8 \dagger$ \\
\hline Kokain & $18 \%$ & $7 \%$ & n. s. & $1,1( \pm 4,0)$ & $0,5( \pm 1,7)$ & $t=2,0 \dagger$ \\
\hline Amphetamine & $5 \%$ & $4 \%$ & n. s. & $0,1( \pm 0,5)$ & $0,6( \pm 3,3)$ & n. s. \\
\hline Cannabis & $53 \%$ & $30 \%$ & n. s. & $8,2( \pm 11,2)$ & $7,2( \pm 10,6)$ & $\mathrm{t}=2,0 \dagger$ \\
\hline Halluzinogene & $5 \%$ & $1 \%$ & n. s. & $0,2( \pm 1,4)$ & $0,0( \pm 0,1)$ & n. s. \\
\hline mehr als 1 Substanz/Tag & $34 \%$ & $16 \%$ & 3) $\dagger$ & $4,2( \pm 8,6)$ & $3,0( \pm 7,6)$ & $t=1,9 \dagger$ \\
\hline i. v.-Konsum & $31 \%$ & $9 \%$ & $\chi^{2}=8,0^{* *}$ & $5,0( \pm 9,8)$ & $0,8( \pm 2,4)$ & $\mathrm{t}=4,1^{* *}$ \\
\hline
\end{tabular}

1) McNemar-Test $(\mathrm{n}=92):{ }^{* *} \mathrm{p}<0.01$

2) $\mathrm{t}$-Test für abhängige Stichproben $(\mathrm{n}=92)$ : $\dagger \mathrm{p}<0.10 ;{ }^{*} \mathrm{p}<0.05 ;{ }^{* *} \mathrm{p}<0.01$

3) exakte Signifikanz basierend auf Binomialverteilung $(n=92)$ : $\dagger p<0.10 ;{ }^{*} p<0.05$ 
ein kontinuierlicher Rückgang der Entzugssymptomatik (Repeated measures ANOVA: Pillai's trace $=0,37, \mathrm{df}=67, \mathrm{p}<0,001$ ), der sich nach 5 Wochen Behandlungsdauer $\left(\mathrm{zu} \mathrm{T}_{2}\right.$ ) etwas abschwächte. Auch aus ärztlicher Sicht ging die Entzugssymptomatik im Verlauf der Studie zurück (Repeated measures ANOVA: Pillai's trace $=0,25, \mathrm{df}=97, \mathrm{p}<0,001$ ), der Effekt war aber insgesamt nicht so deutlich wie bei den Patienteneinschätzungen. Dies könnte jedoch darauf zurückzuführen sein, dass die Einschätzungen der Ärzte erst an $\mathrm{T}_{1}$ einsetzten.

\section{Einflussfaktoren des Behandlungserfolgs}

Was unterscheidet Patienten, die die Entzugsbehandlung erfolgreich beendet haben, von jenen, denen es nicht gelungen ist, im Verlauf der 12-wöchigen Behandlung den Opiatkonsum einzustellen? Zu diesem Zweck werden diese Patientengruppen miteinander verglichen. Der Vergleich erfolgt nicht anhand der Ärzteangaben zum Abbruch oder regulären Ende der Behandlung (siehe oben), da auch unter den regulären Beendern noch Patienten Opiate konsumieren bzw. unter den Behandlungsabbrechern Patienten mit dem Opiatkonsum aufgehört haben. Als Vergleichsvariable wird das Ergebnis der letzten Urinkontrolle (zu $\mathrm{T}_{4 / \mathrm{zz}}$ ) bei 114 Patienten herangezogen. Wie bereits dargestellt, lassen sich bei $72 \%$ der Patienten zum Ende der Entzugsbehandlung kein Methadon oder andere Opiate mehr im Urin nachweisen. Sieht man vom Alter ab - die Patienten, die die Entzugsbehandlung erfolgreich beendeten, sind mit 33,2 Jahren im Durchschnitt fünf Jahre älter als die anderen (28,3 Jahre) -, bestehen hinsichtlich der Patientenmerkmale und der Lebenssituation zu Behandlungsbeginn keine signifikanten Unterschiede zwischen den Gruppen. Auch in der Behandlungsdauer gibt es keine bedeutsamen Unterschiede. Legt man die Patientenaussagen zugrunde, gebrauchten die erfolgreichen Beender zu Behandlungsbeginn an durchschnittlich 8,9 Tagen, die nicht erfolgreichen Patienten an 9,9 Tagen innerhalb des letzten Monats Heroin. Bis zum letzten Untersuchungszeitpunkt reduzierte sich dieser Konsum erheblich: auf durchschnittlich einen Tag pro Monat bei den Erfolgreichen sowie auf - definitionsbedingt höhere - 3,3 Tage bei den anderen Patienten. Ähnlich verhält es sich beim Vergleich des Konsums von anderen Drogen wie Kokain, Benzodiazepinen und Alkohol. Die erfolgreichen Beender gebrauchen zum Ende etwas mehr Alkohol und Benzodiazepine, nehmen aber weniger Kokain als die Patienten, die nicht von Opiaten entzogen haben. Hinsichtlich der Veränderung während der Behandlung ist jedoch bei beiden Gruppen ein Rückgang des Konsums dieser Substanzen festzustellen. Ähnlich verhält es sich mit der Anzahl von opiatbedingten Nebenwirkungen und psychischen Symptomen wie Depressivität und Ängstlichkeit. Sowohl unter den erfolgreichen Entzugspatienten als auch unter den (außer Buprenorphin) weiterhin Opiate konsumierenden Patienten gehen die Nebenwirkungen zurück und es kommt zu einer deutlichen Abnahme von depressiven und Angstsymptomen. Signifikante Unterschiede zwischen den beiden Gruppen bestehen aber weder zu Behandlungsbeginn noch zum Zeitpunkt der letzten Untersuchung. Damit können, abgesehen vom Alter, bedeutsame Einflussfaktoren bzw. Variablen, die einen möglichen Erfolg der Entzugsbehandlung begünstigen würden, nicht nachgewiesen werden.
Diskussion

In einer naturalistischen Anwendungsbeobachtung über zwölf Wochen wurde der Einsatz von Buprenorphin im Rahmen der ambulanten Entzugsbehandlung bei niedergelassenen Ärzten untersucht [23]. Von 314 insgesamt in die Untersuchungen einbezogenen Patienten wollten 148 mit Hilfe von Buprenorphin einen Drogenentzug durchführen (47\%). Damit scheint sich der Einsatz von Buprenorphin für die ambulante Entzugsbehandlung aus Sicht der an der AWB teilnehmenden Ärzte besonders anzubieten. Der mit 31\% vergleichsweise hohe Anteil an Patienten, die die Behandlung selbst bezahlen, deutet auf eine hohe Motivation unter den Betroffenen hin, den Gebrauch von Opiaten aufzugeben. Ein weiterer Erklärungsgrund für den hohen Anteil an Selbstzahlern dürfte in der insgesamt relativ guten Gesamtsituation der Entzugspatienten liegen. Sieht man davon ab, dass unter den Entzugspatienten der Anteil an bereits (überwiegend mit Methadon) Substituierten etwas höher ist, unterscheidet sich diese Patientengruppe nicht von denen, die eine Maintenanceoder Überbrückungsbehandlung mit Buprenorphin anstrebten.

Die Situation der Untersuchungsgruppe bei Behandlungsbeginn war gekennzeichnet durch ein insgesamt eher niedriges Konsumniveau, einen relativ guten Gesundheitszustand und meist stabile soziale Verhältnisse. Die Konsumprävalenzen wie auch die Konsumintensitäten waren in der Untersuchungsgruppe, verglichen mit anderen Drogenabhängigen am Beginn einer Entzugsbehandlung, eher niedrig. Damit ist insgesamt eine relativ gute Gesamtsituation der Untersuchungsgruppe zu konstatieren. Dies resultiert z.T. sicherlich daraus, dass Patienten mit niedrigem bis mittlerem Abhängigkeitsniveau explizit als Zielgruppe der Buprenorphinbehandlung ausgewählt wurden. Allerdings könnten auch Selektionseffekte im Vorfeld der Behandlung eine Rolle gespielt haben, die sich aus der Pharmakodynamik von Buprenorphin ergeben. Die Entzugsbehandlung mit einem im Vergleich zu Methadon weniger sedierenden Medikament könnte einerseits gerade für Drogenabhängige in einer eher guten Gesamtsituation besonders attraktiv sein, andererseits auch von den behandelnden Ärzten als besonders geeignet für diese Klientel angesehen werden.

Patienten, die die Entzugsbehandlung regulär beenden, haben gegenüber den (späteren) Abbrechern eine etwas höhere Anfangsdosierung. Dies kann darauf hindeuten, dass die Einstellungsdosis beim buprenorphingestützten Entzug nicht zu niedrig angesetzt werden sollte. Ob bei einigen Patienten bewusst eine niedrigere Anfangsdosis gewählt wurde, um möglichst schnell eine Dosisreduktion herbeizuführen - die dann aber nicht zum gewünschten Effekt führte -, kann im Rahmen dieser Untersuchung nicht geklärt werden. Es fällt zudem auf, dass sich bei regulären Beendern und Abbrechern ein ähnlicher Abdosierungsverlauf bis auf ca. $60 \%$ der Anfangsdosis ergibt. Bevorstehende Behandlungsabbrüche schienen sich entweder nicht anzudeuten oder aber es wurde bei Problemen nicht von der Möglichkeit Gebrauch gemacht, mit einer Erhöhung der Buprenorphindosis zu reagieren, um bevorstehende Behandlungsabbrüche möglicherweise zu vermeiden. 
Im Zentrum der Analyse der Behandlungseffekte stehen die Veränderungen im Konsumverhalten und in der Entzugssymptomatik. Beim Konsumverhalten kam es in der Untersuchungsgruppe zu einem besonders deutlichen Rückgang der Konsumprävalenz und -intensität von Heroin sowie zu einer etwas schwächeren Verringerung des Kokainkonsums. Auch der Konsum nicht verschriebenen Methadons verringerte sich, wie zu erwarten, sehr deutlich. Nach 5 Wochen Behandlung sind bei den Entzugspatienten kaum noch methadon- oder opiatpositive Urinproben vorhanden.

Darüber hinaus kam es im Verlauf der Behandlung mit Buprenorphin zu einer Verringerung der Entzugssymptomatik, sowohl aus Sicht der Patienten als auch nach Einschätzung der Ärzte. Dabei traten analog zu den Veränderungen im Konsumverhalten die größten Veränderungen am Anfang der Behandlung auf und diese stabilisierten sich dann im weiteren Verlauf. Parallel zur Abnahme des Opiatgebrauchs ist die Entzugssymptomatik nach 5 Wochen auf einem sehr niedrigen Niveau angelangt. Allerdings zeigt sich, dass hier die Effekte trotz überwiegend signifikanter Ergebnisse insgesamt eher gering sind. Dies ist in erster Linie auf die bereits bei Behandlungsbeginn relativ geringe Belastung durch Entzugssymptome zurückzuführen, was wiederum darauf hindeutet, dass für die Untersuchungsteilnahme - die Studie begann bereits wenige Tage nach der Zulassung von subutex ${ }^{\circledR}$ zur Substitutionsbehandlung - zunächst Patienten ausgewählt wurden, die aus Sicht des Arztes besonders geeignet erschienen.

Bezogen auf alle 148 Patienten, die die Entzugsbehandlung mit Buprenorphin begonnen haben, lassen sich zum Zeitpunkt der letzten Erhebung bei über der Hälfte keine Opiate oder Methadon mehr nachweisen. Die Intensität des Heroinkonsums sank in dieser Patientengruppe von durchschnittlich 9 auf einen Tag innerhalb des letzten Monats. Auch in der anderen Gruppe, bei der zum Untersuchungsende noch Opiat- oder Methadongebrauch festgestellt werden konnte, ging der Heroinkonsum deutlich zurück. Damit kann der ambulanten Entzugsbehandlung mit Buprenorphin insgesamt eine hohe Wirksamkeit bescheinigt werden. Beim Vergleich der beiden Patientengruppen fällt auf, dass sich, abgesehen vom höheren Alter der erfolgreichen Beender, aus dieser Untersuchung keine den Verlauf der Entzugsbehandlung beeinflussenden Variablen feststellen lassen konnten. Weder ein zu Behandlungsbeginn höherer Opiatkonsum noch stärkere im Behandlungsverlauf auftretende Nebenwirkungen oder Entzugserscheinungen, die ein Scheitern der Entzugsbehandlung wahrscheinlich gemacht hätten, stehen mit dem Behandlungserfolg im Zusammenhang.

Einschränkend muss erwähnt werden, dass nicht von allen Patienten umfassende und valide Informationen zum Verlauf der Behandlung vorliegen (je nach Untersuchungsgegenstand zwischen 92 und 114 Personen von 148). Deshalb können die insgesamt positiven Effekte nur auf Patienten bezogen werden, die über einen bestimmten Zeitraum in der Entzugsbehandlung verbleiben. Ferner unterliegt diese Studie den üblichen Einschränkungen naturalistischer Verlaufsstudien wie z.B. dem Fehlen einer Kontrollgruppe oder der nicht vorhandenen Nachuntersuchung von Drop-outs. Dies schränkt die Generalisierbarkeit der beobachteten Befunde ein. Der deutliche Konsumrückgang auch bei Patienten, denen kein Behandlungserfolg innerhalb der 12-wöchigen Unter- suchungsperiode bescheinigt werden konnte, deutet jedoch darauf hin, dass es für viele Opiatabhängige nützlich sein kann, eine ambulante Entzugsbehandlung mit Buprenorphin anzubieten.

\section{Literatur}

${ }^{1}$ Leune J. Zahlen, Fakten und Trends im Hilfesystem. In: Deutsche Hauptstelle gegen die Suchtgefahren e.V (Hrsg). Jahrbuch Sucht 2002. Geesthacht/Neuland 2001; 135-150

${ }^{2}$ EBDD. Jahresbericht über den Stand der Drogenproblematik in der Europäischen Union. Amt für amtliche Veröffentlichungen der Europäischen Gemeinschaften. Luxemburg 2001

3 Auriacombe M, Franques P, Tignol J. Deaths attributable to methadone vs buprenorphine in France. Jama 2001; 285 (1): 45

${ }^{4}$ Thirion X, Micallef J, Barrau K et al. Oberservation of Psychoactive Substance Consumption: Methods and Results of the french OPPIDUM Programme. European Addiction Research 2001; 7: 32 - 37

${ }^{5}$ De Ducla M, Gagnon A, Mucchielli A et al. Comparison of high dose buprenorphine treatments of opiate dependent outpatients in four healthcare networks. Annales de medecine interne 2000; 151 (Suppl. B): B9-15

${ }^{6}$ Dole VP, Nyswander ME. Medical treatment of diacetyl-morphin(heroin)addiction. Jama 1965; 163: 646-650

7 Johnson RE, Jaffe JH, Fudala PJ. A controlled trial of bruprenorphine treatment for opioid dependence. Journal of American Medical Association 1992; 267: 2750-2755

${ }^{8}$ Kosten TR, Schottenfield RS, Ziedonis T et al. Buprenorphine versus methadone for opioid dependence. Journal of Nervous and Mental Disease 1993; 181: 358 - 364

${ }^{9}$ Strain EC, Stitzer ML, Liebson IA et al. Comparison for buprenorphine and methadone in the treatment of opioid dependence. American Journal of Psychiatry 1994; 151: $1025-1030$

10 Ling W, Wesson DR, Charuvastra CH et al. A controlled trial comparing buprenorphine and methadone in opioid dependence. Archives of General Psychiatry 1996; 53: $401-407$

${ }^{11}$ Uehlinger C, Déglon JJ, Livoti S et al. Comparison of buprenorphine and methadone in the treatment of opioid dependence: Swiss multicentre study. European Addiction Research 1998; 4 (Suppl. 1): 13-18

${ }^{12}$ Fischer G, Gombas W, Eder H et al. Buprenorphine versus methadone maintenance for the treatment of opiod dependence. Addiction 1999; 94: $1237-1347$

13 Johnson RE, Chutuape MA, Strain EC et al. A comparison of levomethadyl acetate, buprenorphine, and methadone for opioid dependence. The New England Journal of Medicine 2000; 343: 1290 - 1297

${ }^{14}$ Barnett PG, Rodgers JH, Bloch DA. A meta-analysis comparing buprenorphine to methadone for treatment of opioid dependence. Addiction 2001; 96: 683-690

${ }^{15}$ West SL, O'Neal KK, Graham CW. A meta-analysis comparing the effectiveness of buprenorphine and methadone. Journal of Substance Abuse 2000; 12: $405-414$

${ }^{16}$ Petitjean S, Stohler R, Déglon JJ. Double-blind randomized trail of buprenorphine and methadone in opiate dependence. Drug and Alcohol Dependence 2001; 62: 97-104

${ }^{17}$ Pani PP, Maremmani I, Pirastu R et al. Buprenorphine: a controlled clinical trail in the treatment of opioid dependence. Drug and Alcohol Dependence 2000; 60: 39-50

${ }^{18}$ Diamant K, Fischer G, Schneider C et al. Outpatient opiate detoxification treatment with buprenorphine. Preliminary investigation. European Addiction Research 1998; 4: 198-202

19 Wang RI, Wiesen RL, Lamid S et al. Rating the presence and severity of opiate dependence. Clinical Pharmacology \& Therapeutics 1974; 16 : $653-658$

${ }^{20}$ Resnick BR, Galanter M, Resnick E et al. Buprenorphine treatment of heroin dependence (detoxification and maintenance) in a private practice setting. Journal of Addictive Diseases 2001; 20: 75-83

21 Vignau J. Preliminary assessment of a 10-day rapid detoxification programme using high dosage buprenorphine. European Addiction Research 1998; 4 (Suppl. 1): 29-31

22 Paetzold W, Eronat V, Seifert J et al. Detoxifikation polytoxikomaner Patienten mit Buprenorphin. Auswirkungen auf Affektivität, Angst und Entzugssymptomatik. Nervenarzt 2000; 71: 7: $22-729$ 
${ }^{23}$ Prinzleve M, Verthein U, Farnbacher G et al. Anwendungsbeobachtung zum Einsatz von Subutex ${ }^{\circledR}$ (Buprenorphin) bei Patienten mit Opiatabhängigkeit. Forschungsbericht. Institut für interdisziplinäre Suchtund Drogenforschung ISD. Hamburg 2002

${ }^{24}$ Hautzinger M, Bailer M, Worall $\mathrm{H}$ et al. Beck-Depressions-Inventar (BDI). Bern: Hans-Huber, 1994

25 Laux L, Glanzmann P, Schaffner P et al. STAI. Das State-Trait-Angstinventar. Weinheim: Beltz-Test, 1981
${ }^{26}$ Gossop M. The development of a Short Opiate Withdrawal Scale (SOWS). Addictive Behaviours 1990; 15: 487-490

${ }^{27}$ Türck D, Welsch K. EBIS-Jahresstatistik 1999 der ambulanten Beratungs- und Behandlungsstellen für Suchtkranke in Deutschland. Sucht 2000; 46 (Sonderheft): 7-53 